\title{
Über Kohlensäurebestimmung und -bewertung in Backpulvern.
}

\author{
Erwiderung. \\ Von \\ J. Tillmans und 0 . Heublein. \\ [Eingegangeri am 9. Mär $z$ 1918.]
}

In der unter obigem Titel erschienenen Arbeit haben H. Schellbach und Fr. Bodinus ${ }^{1}$ ) ein neues Verfahren der Kohlensäurebestimmung in Backpulvern bekanntgegeben. Sie haben ferner an einigen vor uns vor kurzem ${ }^{2}$ ) aufgestellten Forderungen über die Beschaffenheit von Backpulver Kritik geübt und haben auch einige neue Ansichten über Backpulver ausgesprochen.

Was die Beurteilung der neuen Untersuchungsmethode angeht, so können wir uns kurz fassen. Sie ist ziemlich umständlich und mit einer Reihe von Fehlerquellen behaftet. Es gelingt z. B. bekanntlich nicht, die Kohlensäure durch kurzes Aufkochen aus einer derartigen Apparatur restlos herauszubringen. Es dürften nicht unbeträchtliche Reste zurückbleiben. Wir brauchen in dieser Hinsicht ja nur an die Handbabung des Geibler'schen Kohlensäurebestimmungsapparates zu erinnern. Längeres Luftdurchsaugen nach beendeter Erhitzung ist hier vorgeschrieben. Immerhin mag aber die Methode geeignet sein, den Gesamttrieb von Backpulvern mit einiger Genauigkeit zu ermitteln, vorausgesetzt, daß recbt sorgfältig gearbeitet wird; unmöglich ist es aber mit Hilfe der angegebenen Methode den Vortrieb zu bestimmen. Es ist selbstverständlich, daß, wenn man so verfährt, wie Schellbach und Bodinus für die Ermittelung eines starken Vortriebes angeben, große Mengen von Kohlensäure

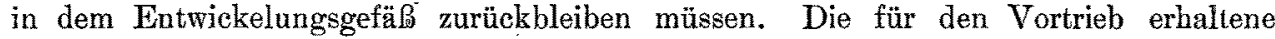
Zahl muß ganz falsch werden. Es ist richtig, daß auch nach" unserem Vertahren der Vortrieb nicht mit derselben Genauigkeit bestimmt werden kann, wie der Gesamttrieb. Das liegt aber nicht an dem Verfahren, sondern an dem Wesen des Vortriebes bezw. Nachtriebes. Die Grenzen von Vor- und Nachtrieb sind flüssig. Man kann deshalb bei Doppelbestimmungen das eine Mal etwas mehr Vortrieb und entsprechend weniger Nachtrieb finden, als das andere Mal, wenn man längere Zeit hat einwirken lassen.

Schellbach und Bodinus wollen nun durch Versuche gefunden haben, daß eine Mischung von Kreide und Weinstein, die im Verhältnis von $1 \mathrm{Mol}$ zu $1 \mathrm{Mol}$ gemischt ist, wirksamer sei, als die theoretische Mischung von 1 Mol zu 2 Molen. Sie haben bei der ersten Mischung höbere Triebkraft gefunden. Hieraus müßte folgerichtig geschlossen werden, daß die so beliebte Streckung des Backpulvers mit überschüssigem kohlensaurem Kalk keine Falschung, sondern zweckmäßig ist, da sie einen höheren Trieb liefert.

Schon die einfache theoretische Uberlegung hätte Schellbach und Bodinus sagen müssen, daß ibre Behauptung in dieser allgemeinen Form unmöglich richtig sein kann. Denken wir uns die beiden. Mischungen in gleichen Mengen Wasser aufgeschwemmt und aufgekocht bezw. abgedampft, so haben wir in der theoretischen

1) Diese Zeitschrift 1918, 35, 236-240. - Die Verfasser hatten die Freundlichkeit, uns einen Durchschlag der Arbeit zur Durchsicht zur Verfügung za stellen.

2) Diese Zeitschrift 1917, 34, 353-374. 
Mischung eine erbeblich größere Weinsäurekonzentration. Nach chemischen Regeln muß dieser größeren Säurekonzentration auch eine größere Umsetzung entsprechen.

Für unsere Nachprüfung mischten wir verschieden feines Material von kohlensaurem Kalk mit Weinstein im Verhältnis von $1 \mathrm{Mol}+1 \mathrm{Mol}$ und $1 \mathrm{Mol}+2 \mathrm{Mol}$. Wir verwendeten präcipitierten kohlensauren Kalk, feingemablene Kreide und fein. gepulverten Marmor. In diesen Mischungen wurde nun jedesmal der gesamte Trieb oder, wie Schellbach und Bodinus sagen, die aktive Kohlensäure bestimmt. Zu dem Zwecke kochten wir 0,5 g Substanz nach dem von uns angegebenen 'Verfahren eine Zeitlang mit Wasser auf und dampften es dann ab. Der Rúckstand wurde in unseren Apparat befôrdert und die in ihm noch vorhandene Kohlensäure bestimmt. Ferner wurde in $0,5 \mathrm{~g}$ aller Mischungen sofort die vorhandene Gesamt-Koblensäure im Apparate ermittelt. Die Differenz ist die aktive Kohlensäure. In den Spalten der nachstehenden Tabellen, die mit dem Wort "theoretisch" überschrieben sind, sind diejenigen Zahlen eingetragen, welche sich rechnerisch ermitteln unter der Annahme völliger Umsetzung des vorhandenen Weinsteins mit dem kohlensauren Kalk und der weiteren Annahme, daß die verwendeten Chemikalien $100 \%$.ig sind.

Bei dem Versuche ergaben sich, auf $1 \mathrm{~g}$ umgerechnet, folgende Zahlen:

\begin{tabular}{|c|c|c|c|c|c|c|c|}
\hline \multirow{2}{*}{\multicolumn{2}{|c|}{ Je $1 \mathrm{~g}$ Mischung }} & \multicolumn{2}{|c|}{$\begin{array}{c}\text { Gesamt-Kohlensäture } \\
\text { ccm }\end{array}$} & \multicolumn{2}{|c|}{$\begin{array}{l}\text { Gesamttrieb (aktive } \\
\text { Kohlensäure) cem } \\
\text { durch Kochen und }\end{array}$} & \multicolumn{2}{|c|}{$\begin{array}{c}\text { Unwirksame } \\
\text { Kohlensäure ccm } \\
\text { dbdampfen ermittelt }\end{array}$} \\
\hline & & gefunden & theoretiseh & gefundon & theoretiseh & gefunden & theoretiseh \\
\hline $\begin{array}{c}\text { Präeipitierter } \\
\text { koblensaurer } \\
\text { Kalk und } \\
\text { Weinstein }\end{array}$ & $\begin{array}{l}1+2 \text { Mole } \\
1+1 \text { Mole }\end{array}$ & $\begin{array}{l}48,0 \\
74,0\end{array}$ & $\begin{array}{l}47,0 \\
77,6\end{array}$ & $\begin{array}{l}46,0 \\
39,0\end{array}$ & $\begin{array}{l}47,0 \\
38,8\end{array}$ & $\begin{array}{r}2,0 \\
35,0\end{array}$ & $\begin{array}{c}0 \\
38,8\end{array}$ \\
\hline $\begin{array}{c}\text { Gemahlene } \\
\text { Kreide und } \\
\text { Weinstein }\end{array}$ & $\begin{array}{l}1+2 \text { Mole } \\
1+1 \text { Mole }\end{array}$ & $\begin{array}{l}44,0 \\
74,5\end{array}$ & $\begin{array}{l}47,0 \\
77,6\end{array}$ & $\begin{array}{l}43,5 \\
37,0\end{array}$ & $\begin{array}{l}47,0 \\
\mathbf{3} 8,8\end{array}$ & $\begin{array}{r}0,5 \\
37,5\end{array}$ & $\begin{array}{c}0 \\
38,8\end{array}$ \\
\hline $\begin{array}{l}\text { Gepulverter } \\
\text { Marmor und } \\
\text { Weinstein }\end{array}$ & $\begin{array}{l}1+2 \text { Mole } \\
1+1 \text { Mole }\end{array}$ & $\begin{array}{l}46,0 \\
73,5\end{array}$ & $\begin{array}{l}47,0 \\
77,6\end{array}$ & $\begin{array}{l}27,8 \\
30,7\end{array}$ & $\begin{array}{l}47,0 \\
38,8\end{array}$ & $\begin{array}{l}20,2 \\
42,8\end{array}$ & $\begin{array}{c}0 \\
38,8\end{array}$ \\
\hline
\end{tabular}

Da Schellbach und Bodinus für die Ermittelung der aktiven Kohlensäure nur sehr kurze Zeit kochen und ferner naturgemäß nicht abdampfen können, so führten wir eine zweite Versuchsreihe in ganz derselben Weise nur mit dem Unterschiede aus, daß wir das im Wasser aufgeschwemmte Pulver jedesmal genaụ 1/4 Minute lang aufkochten; darauf wurde sofort abgekühlt und im Apparate die noch nicht umgesetzte Kohlensäure ermittelt. Diese Rest-Kohlensäure von der früher bestimmten Gesamt-Kohlensäure wiederum abgezogen, ergibt den Gesamttrieb. Dieser Versuch lieferte das nachstehende Frgebnis: 


\begin{tabular}{|c|c|c|c|c|c|c|c|}
\hline \multirow{2}{*}{\multicolumn{2}{|c|}{ Je 1 g Mischung }} & \multicolumn{2}{|c|}{$\begin{array}{c}\text { Gesamt-Kohlensäure } \\
\text { ecm }\end{array}$} & \multicolumn{2}{|c|}{$\begin{array}{l}\text { Gesamttrieb (aktive } \\
\text { Kohilensäure) ccm } \\
\text { durch } 1 / 4 \text { Min. langes }\end{array}$} & \multicolumn{2}{|c|}{$\begin{array}{c}\text { Unwirksame } \\
\text { Kohlensäure cem } \\
\text { Aufkochen ermittelt }\end{array}$} \\
\hline & & gefunden & theoretisch & gefunden & theoretisch & gefunden & theoretiseh \\
\hline $\begin{array}{l}\text { Präcipitierter } \\
\text { kohlensaurer } \\
\text { Kalk und } \\
\text { Weinstein }\end{array}$ & $\begin{array}{l}1+2 \text { Mole } \\
1+1 \text { Mole }\end{array}$ & $\begin{array}{l}48,0 \\
74,0\end{array}$ & $\begin{array}{l}47,0 \\
77,6\end{array}$ & $\begin{array}{l}47,0 \\
36,0\end{array}$ & $\begin{array}{l}47,0 \\
38,8\end{array}$ & $\begin{array}{r}1.0 \\
38,0\end{array}$ & $\begin{array}{c}0 \\
38,8\end{array}$ \\
\hline $\begin{array}{l}\text { Gemahlene } \\
\text { Kreide und } \\
\text { Weinstein }\end{array}$ & $\begin{array}{l}1+2 \text { Mole } \\
1+1 \text { Mole }\end{array}$ & $\begin{array}{l}44,0 \\
74.5\end{array}$ & $\begin{array}{l}47,0 \\
77,6\end{array}$ & $\begin{array}{l}42,0 \\
38,5\end{array}$ & $\begin{array}{l}47,0 \\
38,8\end{array}$ & $\begin{array}{r}2,0 \\
35,0\end{array}$ & $\begin{array}{c}0 \\
38,8\end{array}$ \\
\hline $\begin{array}{l}\text { Gepulverter } \\
\text { Marmor und } \\
\text { Weinstein }\end{array}$ & $\begin{array}{l}1+2 \text { Mole } \\
1+1 \text { Mole }\end{array}$ & $\begin{array}{l}46,0 \\
73,5\end{array}$ & $\begin{array}{l}47.0 \\
77,6\end{array}$ & $\begin{array}{l}25,0 \\
26,5\end{array}$ & $\begin{array}{l}47,0 \\
38,8\end{array}$ & $\begin{array}{l}21,0 \\
47,0\end{array}$ & $\begin{array}{c}0 \\
38,8\end{array}$ \\
\hline
\end{tabular}

Die Versuche ergeben zunächst, daß es praktisch ziemlich gleichgültig ist, ob man nur $1 / 4$ Minute lang aufkocht oder aufkocht und abdampft. Die erhaltenen Zahlen sind praktisch als identisch $\mathrm{zu}$ betrachten.

Die Versuche geben aber nun in sehr interessanter Weise Aufschluß über die Ursache des seltsamen Befundes von Schellbach und Bodinus. Der präcipitierte kohlensaure Kalk und die fein gemahlene Kreide sind jedesmal restlos umgesetzt. Jedesmal ist etwa diejenige Menge Kohlensäure entwichen, welche nach dem vorhandenen Weinsteingehalt gebildet werden kann. Beim gepulverten Marmor werden aber die Verbältnisse anders. Hier bleiben beträchtliche Mengen unzersetzt. Bei der Zersetzung des Präcipitates sowohl, wie der gemablenen Kreide ist keine Rede davon, daß die Mischung $1+1$ wirksamer sei, als die Mischung $1+2$. Durchaus im Einklang mit der Theorie sind vielmehr bei der Mischung $1+2$ stets absolut größere Mengen an Kohlensäuregas entbunden worden, als bei der Mischung $1+1$. Beim Marmorpulver dreht sich aber das Verhältnis um. Hier fanden wir ebenfalls, wie Schellbach und Bodinus, daß die Mischung $1+1$ wirksamer war.

Die Ursache liegt auf der Hand. Sie ist in der Feinheit des Materials begründet. Es ist eine bekannte Tatsache, daß die Schnelligkeit der Lösung von kohlensaurem Kalk, wie überhaupt von mineralischen Stoffen in Säuren, von der Korngröße abhängig ist. Präcipitat und gemahlene Kreide sind fein genug, um in der zur Verfügung stehenden Zeit vom Weinstein restlos gelöst zu werden. Bei dem Marmorpulver ist dies aber offenbar noch nicht der Fall. Ein derartiges Pulver besteht also aus einem feineren und einem gröberen Anteil. Nur der feine Anteil setzt sich in der Versuchszeit mit dem Weinstein um, während der gröbere Anteil mehr oder weniger ungelöst bleibt. In einer kalkreicheren Mischung, wie es die Mischung $1+1$ ist, sind nun natürlich absolut mehr feine Kalkteilchen vorhanden, als in einer kalkärmeren Mischung, z. B. der Mischung $1+2$. In der ersten Mischung mul also auch mehr Kalk umgesetzt werden können. Der Befund von Schellbach und Bodinus, daß die Streckung mit kohlensaurem Kalk einen größeren Trieb veranlasse, ist also nur richtig für verhältnismäßig grobes Material. Mit dieser Feststellung entfällt aber jedes Interesse an dem Befunde der Verfasser. Vor kurzem sind die unter 
Beteiligung zahlreicher Fachgenossen aufgestellten Richtlinien über Ersatzlebensmittel des Kaiserlichen Gesundheitsamtes bekanntgegeben worden ${ }^{1}$ ). In diesen Richtlinien ist die Benutzung von kohlensaurem Kalk für Backpulver überhaupt verboten worden, was sehr zu begrüßen ist. Nur reinster, präcipitierter kohlensaurer Kalk, also allerfeinstes Material, ist zur Verdünnung der Mischung bis zu $20 \%$ gestattet, solange Mehl für diesen Zweck nicht frei gegeben wird. Indessen glauben wir, daß såmtliche Fachgenossen mit Einschluß von Schellbach und Bodinus auch ohne die Richtlinien des Kaiserlichen Gesundheitsamtes mit uns darin einer Meinung sein werden, daß für Backpulver selbstverständlich nur allerfeinstes Material verwendet werden darf.

Die wichtigste Behauptung von Schellbach und Bodinus besagt nun, daB die von uns aufgestellte Forderung, daß ein Backpulver, welehes für 1 Pfund Mehl Verwendung finden soll, wenigstens $1000 \mathrm{ccm}$ Gas entwickeln müsse, unhaltbar sei. Sie schreiben, daß wir diese Forderung "kategorisch" gestellt hätten. Das ist unrichtig, wir sind vielmehr nach langen Überlegungen und praktischen Versuchen zu der Forderung gelangt. Es mag nützlich sein, kurz daran zu erinnern, wie wir zur Aufstellung dieser Zahl gekommen sind.

Zunächst haben wir aus der Literatur festgestellt, welche Zusammensetzung die normalen Friedensbackpulver hatten. Wir fanden dabei, daf ein normales Friedensbackpulver ungefähr $1136 \mathrm{cem}$ Trieb besessen hat. Backpulver ist ein Ersatz für Hefe. Wir haben deshalb ferner berechnet, wie viel Gas bei normalem Backen mit der üblichen Menge Hefe ungefäbr entwickelt wird. Dabej ergab sich eine erheblich größere Gasmenge, als die Friedensbackpulver sie lieferten, nämlich 1600 bis $2667 \mathrm{ccm}$. Die Hauptgrundlage für unsere Zahl 1000 waren aber unsere eigenen Backversuche, die wir mit allen damals üblichen Mischungen unternommen haben.

$\mathrm{Schellbach}$ und Bodin us behaupten, daß sie mit einer Mischung von koblensaurem Kalk und Weinstein im Verhältnis von 1 Mol zu 2 Molen, bei Verwendung von $20 \mathrm{~g}$ auf 1 Pfund Mehl, ein tadelloses Gebäck erhalten hätten, obwohl die vorhandene Menge aktiver Kohlensäure nạ̈ ihren Untersuchungen nur $460 \mathrm{~cm}$ betragen hätte. Dieser Befund ist uns rätselhaft. Nach allen unseren Erfahrungen muß ein derartiges Gebäck vollständig mißraten.

Unsere Mischung von 1 Mol Marmor +2 Mole Weinstein bat nach den oben genannten Untersuchungen einen Gesamttrieb von $516 \mathrm{ccm}$ in $20 \mathrm{~g}$ ergeben; 17,8 $\mathrm{g}$ geben also $460 \mathrm{ccm}$ Trieb. Mit 17,8 g dieser Mischung auf 1 Pfund Mehl haben wir deshalb den Backversuch von Schellbach und Bodinus wiederholt. Wir bereiteten den Teig aus 1 Pfund Weizenmehl Nr. 0, $410 \mathrm{ccm}$ Milch, $100 \mathrm{~g}$ Zucker und $50 \mathrm{~g}$ Butter. Wir benutzten wieder einen Gasbackofen der Firma Junker \& $\mathrm{Ruh}$ in Karlsrube, in dem der Kuchen $1^{1 / 2}$ Stunden lang verblieb.

Der Kuchen ging während des Backens nur in sehr geringem Maße auf. Der Trieb war minimal. Der fertige Kuchen war sehr klein und schwer und von großen Rissen durchzogen. Diese großen Risse entstehen gewöhnlich, wenn kohlensaurer Kalk als Träger der Kohlensäure benutzt wird. Wir werden hierauf noch zurückkommen Das Volumen wurde in derselben Weise gemessen, wie es in unserer früheren Arbeit angegeben ist; es ergab sich zu $1334 \mathrm{ccm}$. Beim Aufschneiden des Kuchens zeigte sich, daß er sehr fest und dicht war und zahlreiche nasse Stellen aufwies. In der

1) Diese Zeitschrift 1918, 35, 183. 
Abbildung Fig. 1 ist die Photographie des Kuchens wiedergegeben. Man erkennt deutlich allenthalben die eigenartigen Risse und die nassen Stellen.

Wir batten ein Backpulver, welches von einer im Bezirke des Bielefelder Untersuchungsamtes liegenden Backpulverfabrik hergestellt wird, beanstandet. Das Backpulver besaß für 1 Pfund Mehl einen Trieb von $535 \mathrm{ccm}$. Es bestand aus Natriumbicarbonat, kohlensaurem Kalk und Weinstein. Weinstein war aber nur in so geringer Menge zugesetzt, daß etwa $38 \%$ Natriumbicarbonat und etwa $23 \%$ koblensaurer Kalk im Überschub verblieben. Herr Dr. Schellbach hat mit ähnlichen Austührungen, wie sie in der in Frage stehenden Arbeit von ihm und Bodinus gemacht sind, die Einstellung des Verfahrens veranlaßt. Er hält ein derartiges Backpulver weder für verfälscht noch für irreführend bezeichnet. Aus den betreffenden Akten haben wir

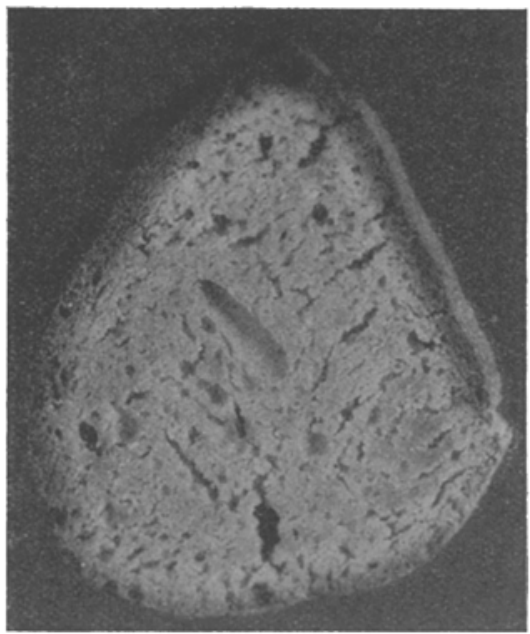

Fig. 1.

Mit kohlensanrem Kalk (gepulvertem Marmor) and Weinstein als Backpulver bereitet. Gesamttrieb $460 \mathrm{ccm}$.

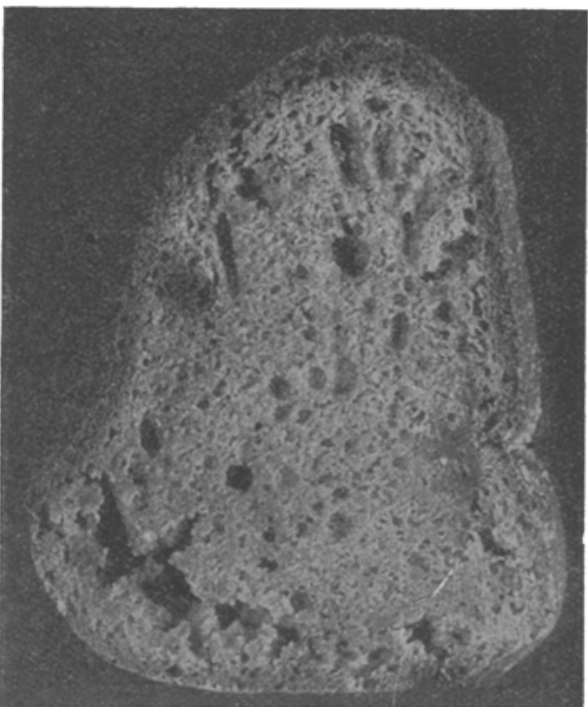

Fig. 2.

Mit $3,34 \mathrm{~g}$ Natriumbicarbonat und $3,34 \mathrm{~g}$ Kreide als Backpulver bereitet.

mit Staunen festgestellt, da mit dieser Auffassung von Herrn Dr. Schellbach aber noch nicht die unterste Grenze seiner Anforderungen an Backpulver erreicht ist. In einem Schreiben an die betreffende Backpulverfabrik bezeichnet Herr Dr. Schellbach eine Mischung von $5 \mathrm{~g}$ Natriumbicarbonat und $5 \mathrm{~g}$ Kreide als ein ausreichendes Backpulver für $1^{1 / 2}$ Pfund Mehl. Die Triebkraft dieser Mischung sei ausreichend und gut. Zu einer Beanstandung läge keine Veranlassung vor.

Solchen Auffassungen gegenüber möchten wir betonen, daß wir die Bezeichnung eines derartigen Gemisches als Backpulver für irreführend halten. Zum Wesen des Backpulvers gehört es, daß neben dem Träger der Kohlensäure ein saurer Bestandteil bezw. ein Salz vorhanden ist, welches die Kohlensäure austreibt. Mit einer Mischung von Natriumbicarbonat und Kreide erbält man nämlich besten Falles einen Kuchen, der unter der Bezeichnung Natronkuchen bekannt ist. Diese Kuchen zeigen einen 
unangenehm laugigen Natrongeschmack und Geruch. Wir befinden uns mit unserer Auffassung, die wir von jeher vertreten haben, in vollster Übereinstimmung mit den neuen Richtlinien des Kaiserlichen Gesundheitsamtes. Auch hier werden dieselben Richtlinien aufgestellt. Es wird ferner dort verlangt, daß nach der Umsetzung kein größerer Überschuß an Natriumbicarbonat als $0,8 \mathrm{~g}$ vorhanden ist. Im übrigen sind wir der Meinung, daß das Publikum solche Mittel wirklich nicht von den Backpulverfabriken hergestellt zu bekommen braucht. Die Kreide ist in der Mischung toter Ballast. Natriumbicarbonat kann sich aber das Publikum einfacher und billiger in jeder Apotheke oder Drogerie selbst kaufen.

Es bedarf unseres Erachtens keines Beweises, daß mit der von Sehell bach und Bodinus empfohlenen Mischung ein minderwertiges Gebäck erhalten werden mub. Trotzdem haben wir in einem Backversuch hierfür noch den Beweis geliefert. Wir mischten 3,34 g Natriumbicarbonat und 3,34 g Kreide und verwendeten dieses als Backpulver für 1 Pfund Mehl Nr. 0, dem sonst dieselben Zusätze gemacht wurden wie bei dem obigen Backversuch. Der Kuchen ging höher auf als der vorige. Sein Volumen betrug $1567 \mathrm{ccm}$. Bei unseren früheren Backversuchen betrug das Volumen eines guten Kuchens aus 1 Pfund Weizenmehl Nr. 0 etwa $1700-2100 \mathrm{ccm}$. Beim Aufschneiden des Kuchens zeigte sich folgendes: Die Krume war leicht gelb verfärbt, was besonders beim Vergleich mit dem früher erwähnten Kuchen auffiel, der rein weiß war. Dieses Gelbwerden der Kuchen zeigt sich stets, wenn überschüssiges Natriumbicarbonat vorhanden ist. Der Geruch und Geschmack war unangenehm laugig. Es war der typische Geruch und Geschmack eines Natronkuchens. Auch in bezug. auf die Lockerung war der Kuchen anormal. Er hatte zahlreiche nasse und nicht aufgegangene Stellen. Die Abbildung Fig. 2 gibt eine Photographie dieses Kuchens wieder.

Es ist zunächst auffallend, daß dieser Kuchen höher aufging, als der vorige. Nur die Hälfte der vorhandenen Bicarbonatkohlensäure könnte eigentlich gewirkt haben. Berechnet man, wie viel das ist, so ergeben sich $444 \mathrm{ccm}$. Das ist also noch weniger als beim vorigen Versuch. Hieraus folgt, daß ohne Zweifel ein Teil der anderen Hälfte der Bicarbonat-Kohlensäure noch durch die Berührung mit dem Mehl umgesetzt worden ist. Die Kuchenmasse zeigte auch, im Wasser aufgeschwemmt, keine alkalische Reaktion gegen Phenolphthalein. Wir haben schon in unserer früheren Arbeit angegeben, daß etwa $3 \mathrm{~g}$ Bicarbonat durch das Mebl neutralisiert werden, ein Befund, der also durch den jetzigen Versuch wiederum bestätigt wird. Es wäre trotzdem sehr falsch, hieraus schließen zu wollen, daf derartige Überschüsse von Bicarbonat unschädlich oder gar nützlich seien. Sie machen sich unter allen Umständen im Kuchen durch den laugigen Geruch und Geschmack bemerkbar. Offenbar handelt es sich dabei um Zersetzungserzeugnisse der Stärke oder der Eiweißkörper ${ }^{1}$ ).

Es kann also nicht die Rede davon sein, daf es sich bei diesen Kuchen um tadellose Gebäcke gehandelt hat; die Kuchen entsprechen vielmehr in beiden Fällen auch nicht sehr mäßigen Ansprüchen. Wir haben darauf verzichtet, den weiteren von Schellbach und Bodinus angegebenen Backversuch mit $660 \mathrm{ccm}$ Trieb ebenfalls zu wiederholen, Auch hier hätte nach allen unseren früheren Backversuchen kein befriedigendes Gebäck herauskommen können.

1) Anmerkung bei der Karrektur: Auf der 15. Hauptversammlung des Vereins Deutscher Nahrungsmittelchemiker (Diese Zeitschrift 1918, 35, 53) hat Geheimrat Her zfeld mitgeteilt, daf Zucker Natriumbicarbonat unter Bildung des Saccharates zu zersetzen vermöge. Möglicherweise handelt es sich also auch um Saccharate. 
Wie sich diese Unterschiede in den Befunden von $\mathrm{Schellbach}$ und Bodinus und uns erklären, vermögen wir nicht zu sagen. Möglicherweise stellen die Autoren auch geringere Anforderungen an die Qualität der Gebäcke als wir.

Wir möchten ferner darauf hinweisen, daß es für das Vorliegen einer Verfälschung durchaus nicht notwendig ist, daß das Backwerk völlig unbrauchbar ist. Ein Backpulver ist verfälscht, wenn die Triebkraft nicht den berechtigten Erwartungen der Konsumenten entspricht. Eine Butter, welche $25 \%$ Wasser enthält, kann, an sich betrachtet, ein gutes Nahrungsmittel sein; trotzdem hat der Bundesrat den Verkauf von Butter, welche mehr als $18 \%$ Wasser enthält, verboten. Eine Butter, die mehr Wasser enthält, ist verfälscht. Ähnlich verhält es sich auch mit den Backpulvern. An sich braucht ein Backpulver mit niedrigem Trieb nicht unbrauchbar zu sein. Viele Hausfrauen haben sich ja schon daran gewöhnt, zwei Päckchen anstatt, wie angegeben, eines auf 1 Pfund Mehl zu verwenden. Trotzdem sind solche Backpulver als verfälscht bezw. irreführend bezeichnet anzusehen.

Im übrigen hat der Nahrungsmittelchemiker unseres Erachtens gar keine Veranlassung, seine Anforderungen an die Triebkraft der Backpulver so zu bemessen, daß sie sich an der Grenze des noch eben Zulässigen bewegen. Es liegt hierfür um so weniger Veranlassung vor, als viele Backpulver, wie wir nachgewiesen haben, sich beim Lagern lebhaft zersetzen.

Wir halten also unsere Angabe, daB ein Backpulver, welches nichtwenigstens $1000 \mathrm{ccm}$ Trieb besitzt, als irreführend bezeichnet anzusehen ist, wenn es nach der Aufschrift für 1 Pfund Mehl Verwendung finden soll, in vollem MaBe aufrecht.

Auch in diesem Punkte befinden wir uns in bester Übereinstimmung mit den neuen Richtlinien des Kaiserlichen Gesundheitsamtes. Diese verlangen sogar noch einen höheren Mindesttrieb, nämlich 2,35 g oder etwa $1200 \mathrm{ccm}$ (genau $1193 \mathrm{ccm}$ ) für 1 Pfund Mehl. Die Richtlinien stellen diese Anforderung allerdings nur für solche Backpulver, bei denen der wirksame Bestandteil Natriumbicarbonat ist. Da sie aber kohlensauren Kalk ganz ausschließen, so bezieht sich diese Forderung auf alle Backpulver mit Ausnahme derjenigen, welche ausschließlich ans Ammoniaksalzen bestehen.

Schellbach und Bodinus halten ein Backpulver mit starkem Vortrieb für ein unzweckmäßiges Backpulver. Dieser Ansicht vermögen wir uns nicht ohne weiteres anzuschließen. Man hat nach unseren Beobachtungen zu unterscheiden zwischen einem guten und schlechten Vortrieb und zwischen einem guten und schlechten Nachtrieb.

Unter einem schlechten Vortrieb ist eine augenblickliche und stürmische Kohlensäureentwickelung beim Vermischen des Backpulvers mit Wasser oder Teig zu verstehen. Setzt die Entwickelung sehr schnell ein, so ist nämlich der größte Teil der Kohlensäure schon verpufft, bevor noch der Teig im Ofen ist. Ein solches Backpulver ist also in der Tat unzweckmäßig. Ein Vortrieb indessen, bei dem die Kohlensäureentwickelung langsam und träge vor sich geht, ist durchaus nicht unwirksam, sondern im Gegenteil recht wirksam, viel wirksamer jedenfalls, als ein schlechter Nachtrieb. Die Friedensbackpulver, welche aus Natriumbicarbonat und Weinstein bestehen, besitzen einen derartigen langsam wirkenden Vortrieb. Die gute Wirkung dieser Friedensbackpulver ist bekannt.

Den Nachtrieb hat man ebenfalls in einen guten und einen schlechten zu unterscheiden, je nach der Temperatur, beỉ welcher die Gasentwickelung vor sich geht. Erfolgt sie schon bei niedrigen. Temperaturgraden, so ist der Nachtrieb ein guter. Er 
lockert nämlich gleichmägig und gut das noch feuchte und dehnungsfähige Gebäck auf. $\mathrm{Zu}$ den Mischungen mit gutem Nachtrieb gehören z. B. die ammoniakhaltigen Backpulver. Die Entwickelung von Ammoniak erfolgt schon bei gelindem Anwärmen, also sofort, nachdem der Kuchen in den Ofen gekommen ist.

Bei dieser Gelegenheit möchten wir einschalten, daß wir gewisse Bedenken gegen die allgemeine Zulassung der Ammoniakbackpulver, wie sie sich aus den Richtlinien ergibt, nicht zu unterdrücken vermögen. Trotz der guten Wirkung, welche das Ammoniak hinsichtlich des Treibens besitzt, weisen doch nach den von uns gemachten Erfahrungen die Ammoniakbackpulver eine Reihe schwerer Mängel auf. Vielfach besitzen die Kuchen im Innern einen deutlichen Geruch nach Ammoniak. Da das Publikum den Ammoniakgeruch nur wahrnimmt an Stellen, wo Abfallstoffe unappetitlicher Natur (Harn, Fäkalien) verwesen, so verbindet es mit diesem Geruch unwillkürlich etwas Unappetitliches. Viele Personen, die empfindlich sind, lehnen daher Kuchen mit Ammoniakgeruch ohne weiteres ab. Durch scharfes Ausbacken kann man freilich gewöhnlich bewirken, dab ein Ammoniakgeruch im Gebäck nicht mehr bemerkbar ist. Wenn indessen viel Ammoniak vorhanden ist, so stellen sich noch andere Unzuträglichkeiten ein. Offenbar bewirkt das Ammoniak in gewissem Maße eine Zersetzung der.Meblbestandteile, sei es des Klebers, sei es der Stärke ${ }^{1}$ ). Jedenfalls haben wir mit Ammoniakbackpulvern hergestellte Kuchen gehabt, die einen widerwärtigen laugigen Geruch und Geschmack aufwiesen, ähnlich wie es bei Kuchen der Fall ist, welche mit Backpulver, die überschüssiges Bicarbonat enthalten, hergestellt sind. Ebenso zeigt sich bei solchen Backpulvern bisweilen die erwähnte gelbe Verfärbung des Mehles.

Ein Nachtrieb ist als schlecht zu beurteilen, wenn er erst bei höherer Temperatur sich entwickelt. Infolge der hohen Temperatur ist der Kuchen schon fester geworden und hat an Ausdehnungsfähigkeit verloren. .Die sich entwickelnde Kohlensäure kann nun nicht mehr in dem Maße, wie beim guten Vortrieb und beim guten Nachtrieb nach allen Beiten hin gleichmäßíg das weiche Gebäck auflockern; es bilden sich vielmehr größere Gasmengen aus, die am Entweichen durch die schon festere Teigbeschaffenheit mehr oder weniger verhindert sind. Die Folge davon ist die Bildung großer Risse im Kuchen an Stelle der bei gutem Vor- und Nachtrieb den ganzen Kuchen durchsetzenden Löcher. Bei derartigem Nachtriebe muß dann natürlich das Gebäck an manchen Stellen feucht bleiben.

$\mathrm{Zu}$ den Backpulvern mit schlechtem Nachtrieb gehören gerade die von Schellbach und Bodin us verwendeten Mischungen von Weinstein mit kohlensaurem Kalk. Die Umsetzung einer derartigen Mischung erfolgt größtenteils erst bei verhältnismäßig hohen Temperaturen. Die Kuchen besitzen denn auch, wie die Abbildung Fig. 1 zeigt, die typischen Eigenschaften des mit schlechtem Nachtrieb erhaltenen Kuchens. Sie zeigen grobe Risse und nasse Stellen.

Sehellbach und Bodinus sind endlich noch der Meinung, daB auch die Säurewirkung des Mehles nicht außer acht zu lassen sei. Backpulver, die überschüssigen kohlensauren Kalk und überschüssiges Natriumbicarbonat enthalten, sollen bei der Berührung mit dem Mehl, ohne daß ein sonstiger saurer Bestandteil vorliegt, beträchtliche Mengen Kohlensäure zu entwickeln vermögen.

Für das Natriumbicarbonat haben wir diese Frage schon oben behandelt. Es ist wahrscheinlich, daß die Mehlbestandteile ${ }^{2}$ ) aus Natriumbicarbonat Kohlensäure in

1) Möglicherweise liegt anch hier wieder eine Zuckerwirknng vor.

$\left.{ }^{2}\right)$ bezw. der Zucker (vergl. Anmerkung 1 S. 262). 
Freiheit zu setzen vermögen. Trotzdem verbietet sich die Anwendung überschüssigen Natrumbicarbonats wegen des schlechten Geruches und Geschmackes, den dadurch der Kuchen annimmt.

Zweifelhafter ist die Entbindung von Kohlensäure aus kohlensaurem Kalk durch die Mehlbestandteile. Die hier in Frage kommenden Säuren sind nämlich sehr schwach, viel schwächer noch als der Weinstein. Sollte indessen trotzdem Kohlensäure entbunden werden, so kann es wohl keinem Zweifel unterliegen, daß dieses nur bei höherer Temperatur geschieht. Es mul sich also bei diesen Kohlensäuremengen um einen schlechten Nachtrieb handeln. Man läßt ihn deshalb am besten auBer Betracht, da er nur eine sehr geringe Wirkung zu entfalten vermag.

Bei dieser Gelegenbeit möchten wir noch eine Unrichtigkeit bezw. Unklarheit beseitigen, welche in unserer vorigen Arbeit über Backpulver vorhanden ist.

Bei der Berechnung der einzelnen Bestandteile wird auf Seite 359 für die Berechnung des überschüssigen Bicarbonats der Faktor 3,75 genannt. Wünscht man aber die gesamte Bicarbonatmenge zu bestimmen, welcher eine Säure zur Umsetzung nicht gegenüberstand, so ist mit dem doppelten Faktor 7,5 zu rechnen. Der Faktor 3,75 würde darauf Rücksicht nehmen, daß die Hälfte der Bicarbonatkohlensäure ja noch als Trieb anzusehen ist. Nur die Hälfte des Bicarbonates, dem eine Säure zur Umsetzung nicht gegenübersteht, würde also hierbei als überschüssig angesehen. Zweckmäßig ist es jedoch, nur mit dem Faktor 7,5 zu rechnen, den Gehalt an überschüssigem Bicarbonat dann aber nicht, wie wir vorschlugen, als Unterrubrik zur unwirksamen Kohlensäure zu nennen, da die Kohlensäure mindestens zur Hälfte aus wirksamer Kohlensäure besteht.

Endlich sei noch angegeben, daß sich bei unseren Untersuchungen herausgestellt hat, daß es zweckmäßiger ist, für die Bestimmung der Kohlensäure im Apparat nicht mit Schwefelsäure zu arbeiten, sondern statt dessen $25 \%$-ige Salzsäure (spez. Gewicht 1,124) zu benutzen. Scheinbar findet durch die starke Schwefelsäure eine Erwärmung statt, die unter Umständen zu hohe Gaswerte ergeben kann.

\section{Zusammenfassung der Ergebnisse.}

Wir fassen die Ergebnisse unserer Arbeit, wie folgt, zusammen:

1. Das von Schellbach und Bodinus bekannt gegebene Verfahren der Bestimmung des Gesamtriebes in Backpulvern ist umständlich und mit manchen Fehlerquellen behaftet; bei sorgfältiger Arbeit mag es aber ausreichen. Mit diesem Verfahren läßt sich aber der Vortrieb nur gänzlich fehlerhaft bestimmen.

2. Die Ansicht von Schellbach und Bodinus, dab eine Mischung von $1 \mathrm{Mol}$ kohlensaurem Kalk $+1 \mathrm{Mol}$ Weinstein praktisch einen größeren Trieb liefere, als die theoretische Mischung von 1 Mol kohlensaurem Kalk und 2 Mol Weinstein, daß also mit anderen Worten die Streckung mit kohlensaurem Kalk zweckmäßig sei, ist irrig. Bei normalem, feingepulvertem Material ist dieses nicht der Fall; nur wenn verhältnismäbig grobes Material verwendet wird, trifft es zu. Da grobes Material für Backpulver aber nicht verwendet werden darf, hat der Befund von Schellbach und Bodin us kein Interesse.

3. Mit $460 \mathrm{ccm}$ Gesamttrieb (auf 1 Pfund Mehl) wollen Schellbach und Bodinus ein tadelloses Gebäck erhalten haben. Wir erhielten mit demselben Trieb einen völlig unbrauchbaren Kuchen, dessen Photographie wiedergegeben ist. Ein weiterer 
Backversuch mit einem Gemisch von Natriumbicarbonat und Kreide lieferte ebenfalls ein schlechtes Gebäck (vergl. Fig. 2).

4. Unsere Forderung nach wenigstens $1000 \mathrm{ccm}$ Gas. für 1 Pfund Mehl ist nach verschiedenen Überlegungen und ausführlichen Backversuchen gewonnen worden. Wir halten sie in vollem Maße aufrecht. Wir befinden uns mit dieser Forderung in voller Übereinstimmung mit den neuen Richtlinien des Kaiserlichen Gesundheitsamtes, indem diese einen noch höheren Mindesttrieb, nämlich rund $1200 \mathrm{ccm}$, fordern.

5. Den Trieb von Backpulvern unterscheidet man zweckmäBig in einen guten und schlechten Vortrieb und in einen guten und schlechten Nachtrieb. Fin schlechter Vortrieb wirkt zu schnell. Ein guter Vortrieb ist eine langsam und allmählich in der Kälte auftretende Kohlensäureentwickelung. Ein guter Nachtrieb ist eine bei niedriger Temperatur vor sich gehende Gasentwickelung, ein schlechter Nachtrieb eine bei höherer Temperatur erfolgende Kohlensäurebildung.

6. Die Säure des Mehls dürte zu schwach sein, um aus Überschüssen von kohlensaurem Kalk Kohlensäure in nennenswerter Menge zu entbinden. In jedem Falle handelt es sich bei der Schwäche der Säure um einen schlechten Nachtrieb.

\section{Typha als Stärkepflanze.}

\section{Von}

Dr. phil. et Mag. pharm. Ludwig Kofler.

Mitteilung aus dem K. K. Pharmakognostischen Universitätsinstitatin Wien.

[Eingegangen am 13. Januar 1918.]

In dem Bestreben, möglichst alle von der Natur gebotenen Nährstoffe für den Menschen unmittelbar oder mittelbar nutzbar zu. machen, richtet sich das Augenmerk vor allem auf wildwachsende Pflanzen, die Kohlenhydrate, Fett und Eiweiß zu liefern vermögen. So wurden im Laufe des Krieges ziemlich alle einheimischen Pflanzen, die als Stärkelieferanten in Betracht kommen, als mensehliche Nahrungsmittel vorgeschlagen und zum Teil auch verwendet. Dabei verfuhr man häufig ohne die genügende Kritik, im günstigsten Falle wurde danñ die Abwesenheit von giftigen Alkaloiden, Glykosiden und Saponinen festgestellt und daraufhin die Pflanze als brauchbares Nahrungsmittel erklärt.

Es besteht nur ein Kriterium für die Unschädlichkeit einer Pflanze und das ist der Versuch am Menschen. Unschädlichkeit beim Verfüttern an Tiere erlaubt noch nicht den gleichen Schluß für den Menschen. Es gibt genügend Beispiele für die verschiedene Wirkung mancher Gifte auf Tier und Mensch, wird doch selbst der Schierling ohne Schaden von den Ziegen gefressen. Wie schlimme Folgen die Außerachtlassung dieser Tatsachen in der Praxis nach sich ziehen kann, zeigt der Fall von Pteridium aquilinum. Die Wurzelstöcke des Adlerfarns wurden in großer Menge gesammelt und sollten als Viehfutter, durch Zusatz zum Brot direkt als menschliche Nahrung und für Biererzeugung verwendet werden. Von berufener Seite wurde zwar die Abwesenheit auf dem üblichen Wege nachweisbarer Giftstoffe zugegeben, aber 